\title{
Correction to "Delay in Diagnosis of Diabetes Mellitus Due to Inaccurate Use of Hemoglobin $\mathrm{A}_{1 \mathrm{C}}$ Levels"
}

Tables 2 and 3 were printed incorrectly in the abovementioned article (Arnold JG, McGowan HJ.J Am Board Fam Med 2007;20(1):93-96). The correct tables appear below. We apologize for these errors, and we regret any confusion or inconvenience they may have caused. (doi: 10.3122/jabfm.2007.03.070057.)

Table 2. Historical Values from Laboratory Examinations for Mrs. J

\begin{tabular}{lcccc}
\hline Date & Fasting Plasma Glucose $(\mathrm{mg} / \mathrm{dL})$ & Hemoglobin $\mathrm{A}_{1 \mathrm{C}}$ & Hemoglobin $(\mathrm{g} / \mathrm{dL})$ & Hematocrit \\
\hline February 1999 & 119 & 0.045 & 9.6 & 0.275 \\
August 1999 & 82 & 0.038 & 12.4 & 11.8 \\
December 2000 & & 0.041 & 10.9 & 0.351 \\
April 2001 & 137 & 0.041 & 10.3 & 0.343 \\
March 2002 & 136 & 0.046 & 11.4 & 0.296 \\
April 2003 & 192 & 0.050 & 10.6 & 0.330 \\
April 2004 & 170 & 0.044 & 0.048 & 0.297 \\
March 2005 & 187 & & &
\end{tabular}

Table 3. $\mathrm{HbA}_{1 \mathrm{c}}$ Correlation with Blood Glucose Readings $^{2}$

\begin{tabular}{lc}
\hline $\mathrm{HbA}_{1 \mathrm{c}}$ & Blood Glucose $(\mathrm{mg} / \mathrm{dl})$ \\
\hline 0.060 & 120 \\
0.070 & 150 \\
0.080 & 180 \\
0.090 & 210 \\
0.10 & 240 \\
0.11 & 270 \\
0.12 & 300 \\
0.13 & 330 \\
0.14 & 360 \\
\hline
\end{tabular}

